\title{
Original Research \\ Evaluation of systemic inflammation in response to remote ischemic preconditioning in patients undergoing transcatheter aortic valve replacement (TAVR)
}

\author{
Kun Zhang ${ }^{1,2,3, \dagger}$, Willi Troeger ${ }^{4,5, \dagger}$, Matthias Kuhn ${ }^{6}$, Stephan Wiedemann ${ }^{7}$, Karim Ibrahim ${ }^{8}$, \\ Christian Pfluecke ${ }^{4,9}$, Krunoslav M. Sveric ${ }^{4}$, Robert Winzer ${ }^{10}$, Dieter Fedders ${ }^{10}$, \\ Tobias F. Ruf ${ }^{11}$, Ruth H. Strasser ${ }^{12}$, Axel Linke ${ }^{4}$, Silvio Quick ${ }^{4,8}$, Felix M. Heidrich ${ }^{4, *}$ \\ ${ }^{1}$ Department of Internal Medicine and Cardiology, Campus Virchow-Klinikum, Charité-Universitätsmedizin Berlin, 13353 Berlin, Germany \\ ${ }^{2}$ Berlin Health Institute, 10178 Berlin, Germany \\ ${ }^{3}$ DZHK (German Centre for Cardiovascular Research), partner site Berlin, 10785 Berlin, Germany \\ ${ }^{4}$ Department of Internal Medicine and Cardiology, Faculty of Medicine Carl Gustav Carus at Technische Universität Dresden, Herzzentrum Dresden, \\ 01307 Dresden, Germany \\ ${ }^{5}$ Department of Anesthesiology and Critical Care Medicine, Medical University of Innsbruck, 6020 Innsbruck, Austria \\ ${ }^{6}$ Institute for Medical Informatics and Biometry, Faculty of Medicine Carl Gustav Carus, Technische Universität Dresden, 01307 Dresden, Germany \\ ${ }^{7}$ Department of Internal Medicine and Cardiology, HELIOS Klinikum Pirna, 01796 Pirna, Germany \\ ${ }^{8}$ Department of Cardiology, Faculty of Medicine Carl Gustav Carus at Technische Universität Dresden, Klinikum Chemnitz, 09116 Chemnitz, Germany \\ ${ }^{9}$ Department of Internal Medicine I, Faculty of Medicine Carl Gustav Carus at Technische Universität Dresden, Klinikum Görlitz, 02828 Görlitz, \\ Germany \\ ${ }^{10}$ Department of Radiology, Faculty of Medicine Carl Gustav Carus at Technische Universität Dresden, Universitätsklinikum Dresden, 01307 Dresden, \\ Germany \\ ${ }^{11}$ Center for Cardiology I, Heart Valve Center Mainz, University Medical Center Mainz, 55131 Mainz, Germany \\ ${ }^{12}$ Faculty of Medicine Carl Gustav Carus, Technische Universität Dresden, 01307 Dresden, Germany \\ *Correspondence: felix.heidrich@tu-dresden.de (Felix M. Heidrich) \\ ${ }^{\dagger}$ These authors contributed equally. \\ Academic Editor: Vincenzo Lionetti \\ Submitted: 8 October 2021 Revised: 2 December 2021 Accepted: 13 December 2021 Published: 14 January 2022
}

\begin{abstract}
Background: Systemic inflammation can occur after transcatheter aortic valve replacement (TAVR) and correlates with adverse outcome. The impact of remote ischemic preconditioning (RIPC) on TAVR associated systemic inflammation is unknown and was focus of this study. Methods: We performed a prospective controlled trial at a single center and included 66 patients treated with remote ischemic preconditioning (RIPC) prior to TAVR, who were matched to a control group by propensity score. RIPC was applied to the upper extremity using a conventional tourniquet. Definition of systemic inflammation was based on leucocyte count, C-reactive protein (CRP), procalcitonin (PCT) and interleukin-6 (IL-6), assessed in the first 5 days following the TAVR procedure. Mortality was determined within 6 months after TAVR. RIPC group and matched control group showed comparable baseline characteristics. Results: Systemic inflammation occurred in $66 \%$ of all patients after TAVR. Overall, survival after 6 months was significantly reduced in patients with systemic inflammation. RIPC, in comparison to control, did not significantly alter the plasma levels of leucocyte count, CRP, PCT or IL-6 within the first 5 days after TAVR. Furthermore, inflammation associated survival after 6 months was not improved by RIPC. Of all peri-interventional variables assessed, only the amount of the applied contrast agent was connected to the occurrence of systemic inflammation. Conclusions: Systemic inflammation frequently occurs after TAVR and leads to increased mortality after 6 months. RIPC neither reduces the incidence of systemic inflammation nor improves inflammation associated patient survival within 6 months.
\end{abstract}

Keywords: Remote ischemic preconditioning; Transcatheter aortic valve replacement; Aortic valve stenosis; Systemic inflammatory response syndrome; Systemic inflammation

\section{Introduction}

Transcatheter aortic valve replacement (TAVR) was first successfully performed in 2002 in a critically ill patient with severe aortic stenosis [1]. Since the past almost 20 years, it has been evolved to a standard procedure for patients at high and intermediate surgical risk, and more recently, its indication is even including patients at low risk [2]. With accumulating experience and advancing tech- nologies, the procedure has become safer with fewer complications. However, adverse events remain [2]. Systemic inflammatory response syndrome (SIRS) occurs in approximately $50 \%$ of patients after the TAVR procedure [3] and is associated with increased morbidity and mortality $[4,5]$. The pathogenesis of systemic inflammation after TAVR is not clear. Sinning et al. [4] observed more rapid ventricular pacing runs and higher post-procedural lactate plasma con- 
centrations in patients with SIRS. This indicates that certain characteristics of the procedure may lead to tissue hypoperfusion/ischemia and are associated with systemic inflammation. In remote ischemic preconditioning (RIPC), alternating intervals of ischemia and reperfusion are applied on a peripheral tissue or organ, which is sought to have protective effects on ischemia and reperfusion injury [6,7]. It has been shown in different experimental settings that inflammatory response can be attenuated by RIPC [8,9].

With this work, we aim to elucidate the impact of RIPC on systemic inflammation in patients undergoing TAVR.

\section{Materials and methods}

\subsection{Study design and patient enrolment}

We performed a prospective controlled trial recruiting patients at Heart Center Dresden from February 2014 to December 2016. Data on control group patients (nonRIPC group) were acquired retrospectively. Decision for TAVR was made in the interdisciplinary Heart Team based on current guideline recommendations [10]. Exclusion criteria were second intervention ("valve-in-valve"), cardiogenic shock, use of inotropics, peripheral artery disease with symptoms, thrombosis, terminal kidney failure, active malignancy with a life-expectancy less than one year, or the participation in other trials. In particular, SIRS or sepsis was ruled out before intervention. Written informed consent was obtained.

\subsection{Study endpoints}

(1) Occurrence of systemic inflammation following TAVR in RIPC vs. non-RIPC patients. Systemic inflammation was defined by the presence of at least one of the following criteria within the first five post-interventional days: leucocyte count $>12000 / \mathrm{nL}$ or $<4000 / \mathrm{nL}, \mathrm{CRP}>80$ $\mathrm{mg} / \mathrm{dL}$, PCT $>0.4 \mathrm{ng} / \mathrm{mL}$, or IL-6 $>80 \mathrm{pg} / \mathrm{mL}[11,12]$.

(2) Mortality assessment at 6 months after TAVR (all patients included, presence of systemic inflammation).

(3) Mortality assessment at 6 months after TAVR (patients with systemic inflammation only, RIPC status).

(4) TAVR associated systemic inflammation: Impact of peri-interventional parameters.

\subsection{Procedure of TAVR and RIPC}

Aortic valves were implanted through the transfemoral approach and under general anesthesia, as previously described [13]. With the induction of anesthesia, RIPC was conducted with three cycles of ischemia followed byreperfusion for five minutes each. A pressure of 20-30 mmHg above the systolic arterial pressure was applied by using a standard blood-pressure-manometer cuff (Boso, Jungingen, Germany). Efficacy was clinically assessed: pulselessness of the radial artery, acrocyanosis and reactive hyperemia. The start of the TAVR intervention was less than 30 minutes after finishing RIPC. Patients received either a self-expandable CoreValve Evolut R (Medtronic, Minneapolis, MN, USA) $(\mathrm{n}=44)$ or a balloon-expandable Sapien XT/Sapien 3 (Edwards Lifesciences Inc., Irvine, CA, USA) $(n=22)$ valve. Imeron 350 (Bracco S.p.A., Milan, Italy) was used as contrast agent.

\subsection{Laboratory parameters}

Venous blood samples were taken at the day of admission and the first five days after the TAVR procedure in the morning at rest. Inflammatory parameters including leucocyte count, C-reactive protein (CRP), procalcitonin (PCT), and interleukin-6 (IL-6) were measured.

\subsection{Statistical analysis}

Patients receiving RIPC were matched to a control group patients by propensity score, i.e., using the method of nearest-neighbour by the MatchIt-package [14] in " $R$ " (The R Foundation for Statistical Computing, Vienna, Austria). Patients were matched according to following criteria: age, sex, left ventricular ejection fraction, presence of relevant coronary artery disease (CAD), creatinine concentration, type of implanted bioprosthesis, number of periprocedural pacing runs, and volume of contrast agent used during the procedure. All statistical tests were run by Statistical Package for Social Sciences, version 23 (SPSS Inc., IBM, Armonk, NY, USA). Parametric data are presented as mean \pm standard deviation (SD), while non-parametric data are presented as median [inter-quartile-ranges (IQRs)]. $p$-values $<0.05$ were considered statistically significant. To assess differences in the inflammatory parameters between the RIPC and control groups at different time points, we used a linear mixed model that accounts for individual patient effects and serial autocorrelation. Because the inflammatory parameters were right-skewed, we applied a log-transformation first. Chi-squared test and logistic regression were used for the analysis of influencing factors of systemic inflammation. For survival analyses, KaplanMeier estimates with log-rank test were used and influencing factors on survival were calculated by Cox regression.

\section{Results}

\subsection{Baseline data}

In total, we screened 358 patients for eligibility. After applying the exclusion criteria, 66 RIPC and 66 matched control patients entered statistical analyses. Following propensity score matching, both groups showed no statistically significant differences in baseline characteristics and peri-interventional variables (Tables 1,2). The mean age was around 82 for both groups with an even distribution between male and female patients. The Euro-Score-II to predict surgery associated mortality showed intermediate risk, similar for both groups. The inflammatory parameters, i.e., leucocyte count, CRP, PCT and IL-6, were within the normal range and no patient in either group had fever, nor showed other clinical signs of infection before intervention. 
Table 1. Baseline characteristics.

\begin{tabular}{|c|c|c|c|}
\hline & $\operatorname{RIPC}(n=66)$ & Control $(n=66)$ & $p$-value \\
\hline Age (years) & $82 \pm 4$ & $82 \pm 6$ & 0.96 \\
\hline \multicolumn{4}{|l|}{ Sex } \\
\hline female & $32(49 \%)$ & $31(47 \%)$ & $>0.99$ \\
\hline male & $34(51 \%)$ & $35(53 \%)$ & \\
\hline BMI $\left(\mathrm{kg} / \mathrm{m}^{2}\right)$ & $28.2[25.5 ; 31.2]$ & $27.6[23.8 ; 30.9]$ & 0.17 \\
\hline LVEF (\%) & $55[48 ; 60]$ & $55[45 ; 60]$ & 0.57 \\
\hline \multicolumn{4}{|l|}{ Aortic stenosis } \\
\hline $\operatorname{Vmax}(\mathrm{cm} / \mathrm{s})$ & $404 \pm 94(\mathrm{n}=53)$ & $418 \pm 79(\mathrm{n}=59)$ & 0.57 \\
\hline dpmax (mmHg) & $72[48 ; 85](\mathrm{n}=63)$ & $67[54 ; 89](\mathrm{n}=62)$ & 0.91 \\
\hline dpmean $(\mathrm{mmHg})$ & $40[28 ; 51](n=63)$ & $40[31 ; 54](\mathrm{n}=60)$ & 0.73 \\
\hline CAD & $33(51 \%)$ & $30(46 \%)$ & 0.70 \\
\hline PCI or CABG in history & $26(39 \%)$ & $24(36 \%)$ & 0.86 \\
\hline \multicolumn{4}{|l|}{ Medication } \\
\hline ACE inhibitor or AT1-blocker & $50(76 \%)$ & $42(64 \%)$ & 0.22 \\
\hline$\beta$ blocker & $54(82 \%)$ & $50(76 \%)$ & 0.54 \\
\hline MRA & $6(9 \%)$ & $6(9 \%)$ & $>0.99$ \\
\hline Euro-II-Score & $4.6[2.9 ; 7.6]$ & $4.0[2.7 ; 7.2]$ & 0.73 \\
\hline \multicolumn{4}{|l|}{ Laboratory parameters before TAVR } \\
\hline $\mathrm{TnT}(\mathrm{ng} / \mathrm{L})$ & $27.0[14.8 ; 34.3]$ & $23.0[15.0 ; 51.2]$ & 0.32 \\
\hline creatinine $(\mu \mathrm{mol} / \mathrm{L})$ & $101[79 ; 134]$ & $98[81 ; 116]$ & 0.67 \\
\hline $\operatorname{GFR}\left(\mathrm{mL} / \mathrm{min} / 1.73 \mathrm{~m}^{2}\right)$ & $54 \pm 18$ & $56 \pm 17$ & 0.47 \\
\hline urea $(\mathrm{mmol} / \mathrm{L})$ & $7.1[5.6 ; 10.8]$ & $7.1[5.6 ; 9.6]$ & 0.57 \\
\hline
\end{tabular}

Values are presented as mean $\pm \mathrm{SD}$, median [IQR], or $\mathrm{n}(\%)$. If the number of patients was not $n=66$, it was listed as $n=$ $\mathrm{x}$. RIPC, remote ischemic preconditioning; BMI, body mass index; LVEF, left ventricular ejection fraction; Vmax, maximum velocity; dpmax, maximum pressure gradient; dpmean, mean pressure gradient; $\mathrm{CAD}$, coronary artery disease with $>50 \%$ stenosis; PCI, percutaneous coronary intervention; CABG, coronary artery bypass grafting; ACE, angiotensin converting enzyme; AT1, angiotensin II receptor type 1; MRA, mineralocorticoid receptor antagonist; TnT, high sensitive troponin T; GFR, glomerular filtration rate; TAVR, transcatheter aortic valve replacement.

Table 2. Peri-interventional variables of the TAVR procedure after matching.

\begin{tabular}{lccc}
\hline & RIPC $(\mathrm{n}=66)$ & Control $(\mathrm{n}=66)$ & $p$-value \\
\hline Type of valve & & & \\
Medtronic CoreValve & $44(66.7 \%)$ & $44(66.7 \%)$ & $>0.99$ \\
Edwards Sapien XT/3 & $22(33.3 \%)$ & $22(33.3 \%)$ & \\
Number of dilatations & $2[1 ; 2]$ & $2[1 ; 2]$ & $>0.99$ \\
1 & $18(27.3 \%)$ & $16(24.2 \%)$ & \\
2 & $40(60.6 \%)$ & $43(65.2 \%)$ & \\
3 & $6(9.1 \%)$ & $7(10.6 \%)$ & \\
4 & $2(3.0 \%)$ & 0 & \\
Number of pacing runs & $2[2 ; 3]$ & $2[2 ; 3]$ & 0.99 \\
1 & $40(60.6 \%)$ & $35(53.0 \%)$ & \\
2 & $18(27.3 \%)$ & $25(37.9 \%)$ & \\
3 & $6(9.1 \%)$ & $6(9.1 \%)$ & \\
4 & $2(3.0 \%)$ & 0 & \\
Procedure duration (min) & $54[41 ; 71]$ & $52[42 ; 65]$ & 0.29 \\
Contrast agent amount (mL) & $130[120 ; 150]$ & $130[100 ; 153]$ & 0.23 \\
\hline \multicolumn{4}{l}{ Values are presented as median $[\mathrm{IQR}]$ or n (\%). RIPC, remote ischemic } \\
preconditioning. &
\end{tabular}

\subsection{Systemic inflammation frequently occurs after TAVR and is associated with increased mortality}

Systemic inflammation, which was defined as the occurrence of at least one pathologic inflammatory biomarker following the first 5 post-interventional days after the TAVR procedure, occurred in $66 \%(\mathrm{n}=87)$ of all patients. In the RIPC group, 42 patients (64\%) showed an inflammatory response, in comparison to 45 patients $(68 \%)$ in the control group $(p=0.58)$. Overall, we observed an increase of all four markers of systemic inflammation following the TAVR procedure (Fig. 1). Patients with inflammatory response after the TAVR procedure suffered an increased 6-month mortality ( $p=0.01$, Fig. 2$)$.

\subsection{RIPC has no impact on the occurrence of systemic inflammation following TAVR}

First, the impact of RIPC on parameters of systemic inflammation was analysed. As shown in Fig. 1, none of the four inflammatory parameters assessed in this study significantly differed between the RIPC and control group within the first 5 post-interventional days after TAVR. The comparative numeric values for both groups at baseline, day 2 and day 5 are detailed in Supplementary Table 1. RIPC did not significantly alter the impact on mortality in patients suffering from systemic inflammation ( $p=$ 0.62, Fig. 3). Both groups, patients with TAVR associated systemic inflammation and those without, showed similar baseline characteristics (Supplementary Table 2). 
A

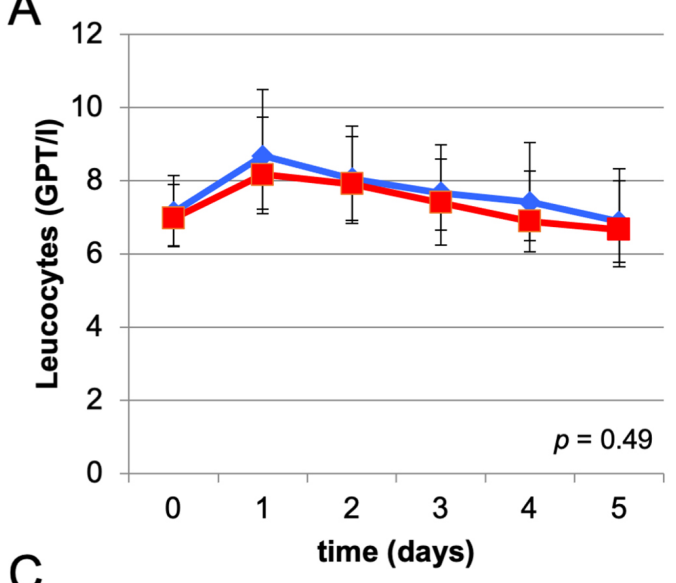

C

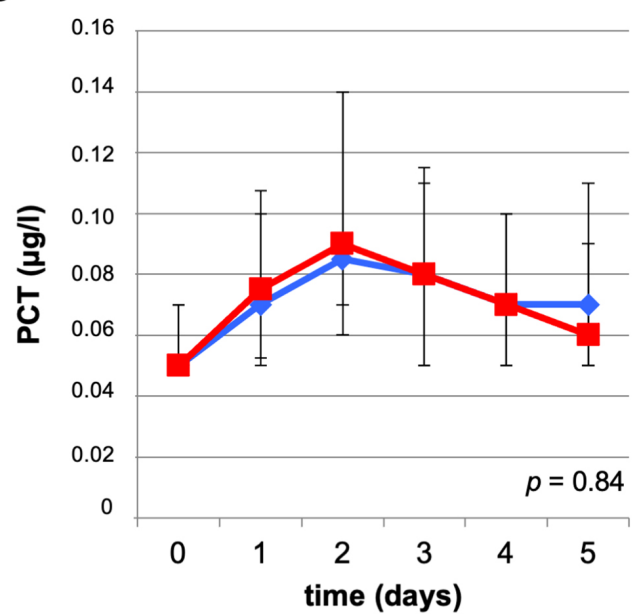

$\mathrm{B}_{100}$

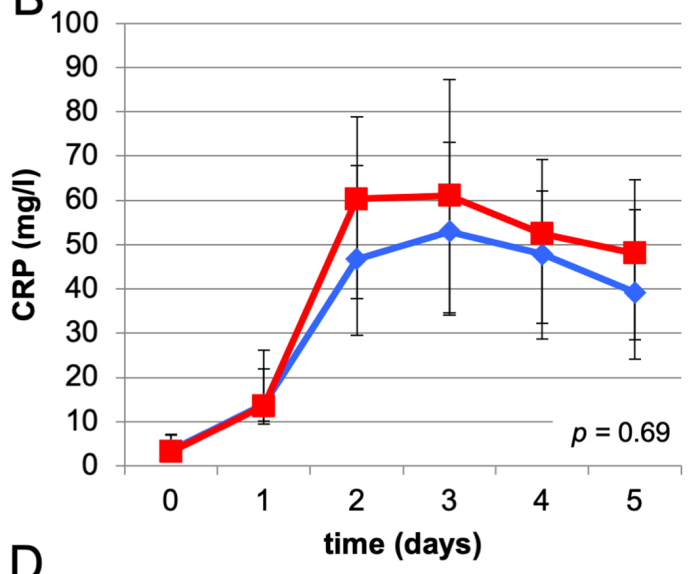

$D_{100}$

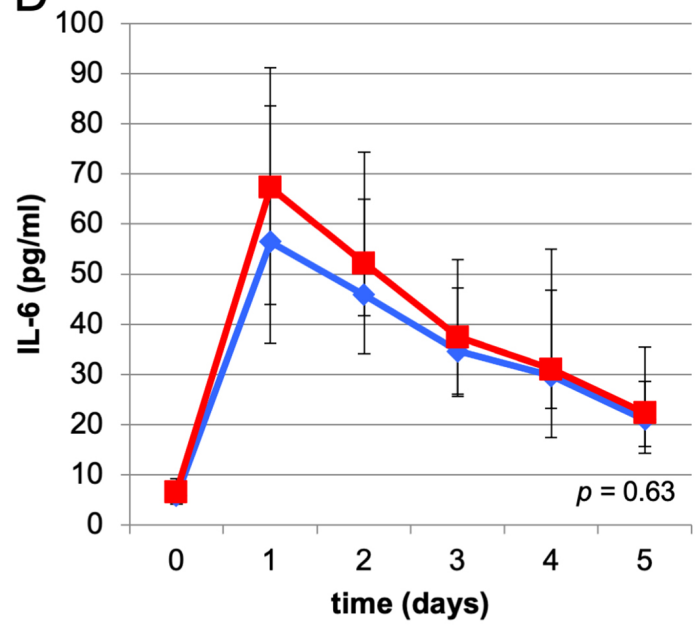

Fig. 1. Time course of inflammatory markers following the TAVR procedure. RIPC with no influence on systemic inflammatory response after TAVR. (A) Leucocyte count. (B) CRP. (C) PCT and (D) IL-6 did not significantly differ between the RIPC and non-RIPC group at any of the time points assessed after TAVR; the respective $p$-value is provided for each parameter. blue solid line - RIPC; red solid line - non-RIPC. CRP, C-reactive protein; PCT, Procalcitonin; IL-6, Interleukin-6.

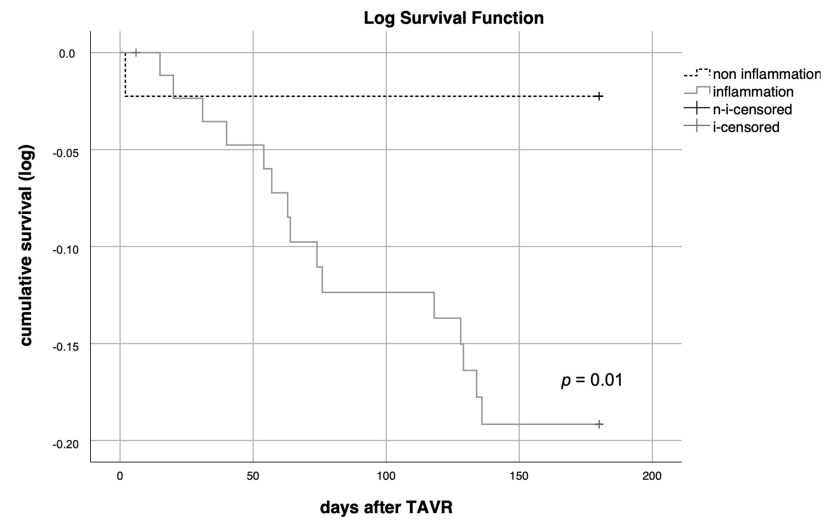

Fig. 2. Negative impact of TAVR associated systemic inflammation on patient survival. Kaplan-Meier-curve showing significantly reduced overall survival in patients with systemic inflammatory response after TAVR within the observation period of 6 months $(p=0.01)$.

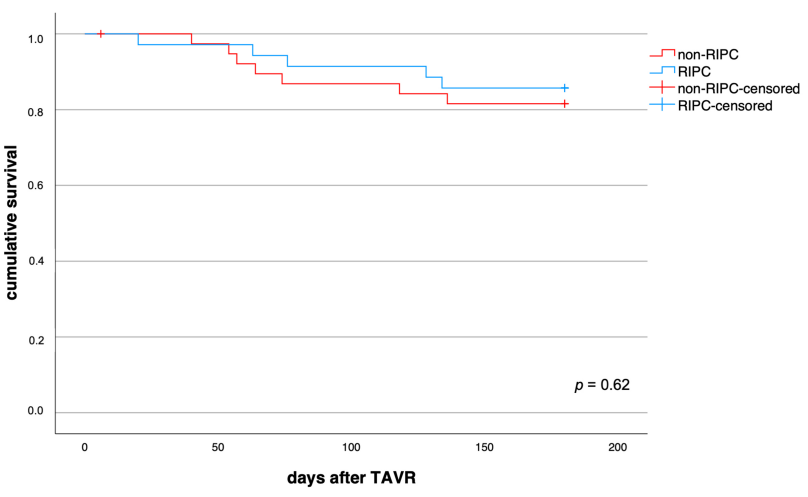

Fig. 3. Impact of RIPC on survival in patients with TAVR associated systemic inflammation. RIPC and non-RIPC displayed comparable impact on 6-month mortality in all patients that suffered from systemic inflammation after TAVR $(p=0.62)$ 
3.4 Peri-interventional amount of contrast agent used is associated with systemic inflammation following TAVR

In a next step, we analysed the impact of periinterventional variables of the TAVR procedure on the occurrence of systemic inflammation. Chi-squared test and regression analysis identified solely the amount of contrast agent used to be associated with the occurrence of systemic inflammation following TAVR $(p=0.02$, Table 3$)$. The number of dilatations, or number of pacing runs, the procedure duration, and the type of implanted bioprosthetic valve showed no association (Table 3 ).

Table 3. Influence of peri-interventional variables on the occurrence of systemic inflammation following TAVR.

\begin{tabular}{lccc}
\hline & $X^{2}(\mathrm{df})$ & Odds ratio $(95 \% \mathrm{CI})$ & $p$-value \\
\hline Type of valve & $3.793(1)$ & - & 0.05 \\
Number of dilatations & $1.983(2)$ & - & 0.37 \\
Number of pacing runs & $2.101(2)$ & - & 0.35 \\
Procedure duration & - & $0.997(0.985-1.008)$ & 0.58 \\
Contrast agent amount & - & $0.989(0.979-0.998)$ & $\mathbf{0 . 0 2}$ \\
\hline
\end{tabular}

Of all peri-interventional variables assessed, Chi-Square-Test and regression analysis identified only the amount of contrast agent used associated with the occurrence of systemic inflammation following TAVR (bold print $p$-value). $X^{2}=$ Pearson chi-squared (degrees of freedom, df), odds ratio ( $95 \%$ confidence interval, CI).

\section{Discussion}

The aim of this study was to assess the effect of RIPC on TAVR associated systemic inflammation. Based on common denominators of systemic inflammation, i.e., leucocyte count, CRP, PCT and IL-6, we estimated the incidence of inflammatory response after TAVR. We showed that systemic inflammation frequently occurs after TAVR and was associated with increased mortality. RIPC, however, did not significantly reduce the occurrence of TAVR associated systemic inflammation. The sole periinterventional variable associated with the occurrence of a significant systemic inflammatory response was the amount of contrast agent used.

The occurrence of a systemic inflammatory response after TAVR has been the focus of investigation in only a few studies and it has been associated with inferior outcomes [4,5,15]. Sinning et al. [4] and Lindman et al. [5], who used a transfemoral approach for TAVR, as was applied in this study, reported an incidence of $40 \%$ and $63 \%$, respectively. In our data, $66 \%$ of all patients suffered from inflammatory response, confirming and emphasizing its high incidence and relevance. Of note, we used a different definition for systemic inflammation, which may account for small discrepancies in the exact numbers. In several other studies examining acute kidney injury after TAVR, a postinterventional increase in leucocyte count with no signs of infection was observed and attributed to a systemic inflam- matory response [16-18]. It was associated with an adverse impact on various outcomes, such as acute kidney injury. In agreement with other studies [4,5], we confirmed that the occurrence of systemic inflammation is associated with significantly increased mortality.

The sufficiency and suitability of biomarkers to assess systemic inflammation can be subject to discussion. Of note, we deliberately avoided to apply the once commonly used SIRS definition, now mostly abandoned because of its low specificity and limited usefulness [19]. Moreover, we did not consider them adequate for our study cohort. The implantation of the bioprosthesis can affect the heart rate as it is oftentimes associated with post interventional atrioventricular blocks. Furthermore, general anesthesia affects the autonomic nervous system with influence on body temperature, heart, and respiratory rates. Therefore, we tried to avoid using potentially confounding factors and instead relied on serological parameters of systemic inflammation that are available in the clinical routine setting. A comparative overview of the commonly known SIRS criteria and this study's definition of systemic inflammation is provided in Supplementary Table 3.

The occurrence and negative impact of an inflammatory response has been well described in patients undergoing cardiac surgery and cardiopulmonary bypass [20, 21]. The predominant mechanisms for triggering this immune response involve the surgical trauma itself and ischemia/reperfusion injury during the procedure [22]. The latter is caused by temporary systemic and organ hypoperfusion resulting in activation of neurohumoral, immunological and inflammatory pathways $[23,24]$. It is therefore plausible that several TAVR procedure associated conditions, which can potentially lead to hypoperfusion and ischemia/reperfusion injury, may contribute to the development of inflammatory response. Sinning et al. [4] identified the number of ventricular pacing runs and major vascular complications as independent predictors of SIRS development. In this study, we identified the amount of the applied contrast agent as the only peri-interventional variable associated with the occurrence of an inflammatory response. Interestingly, the number of dilatations, the number of pacing runs, the procedure duration or the choice of the implanted bioprosthetic valve showed no association with the occurrence of systemic inflammation.

The application of RIPC is described as a promising strategy to avoid adverse outcomes due to ischemia/reperfusion injury [25]. Reversible episodes of ischemia followed by reperfusion are applied in the periphery and is meant to render protective effects through neurohumoral signaling [1]. On the molecular level, it was shown that RIPC leads to suppression of genes encoding key inflammation factors [8]. Mechanical RIPC achieved by repeated inflation and deflation of a blood pressure cuff is an easy-to-use, non-invasive and safe method. In patients suffering from myocardial infarction treated with percutaneous 
coronary intervention and in patients undergoing coronary artery bypass surgery, the application of RIPC was shown to be cardioprotective and beneficial on clinical outcomes [26-28]. Two previous studies on RIPC in TAVR patients did not reveal any beneficial effects with regard to cardioor renoprotection and overall survival $[29,30]$. Given that RIPC is conceived to prevent adverse effects caused by ischemia/reperfusion injury and that systemic inflammation is a possible consequence thereof, it is plausible to assume that RIPC may prevent the occurrence of inflammatory response. This study is the first that examined the effect of RIPC on systemic inflammatory response in patients undergoing TAVR. However, our data indicates that RIPC neither reduces the incidence of systemic inflammation nor improves inflammation associated patient survival within 6 months.

\section{Limitations}

Our study contains some strengths and limitations. The following positive points can be mentioned: The study includes a contemporary cohort of patients undergoing a rather uniform treatment regimen. Baseline characteristics of the compared groups were homogeneous as were the peri-interventional variables. The study is limited by its number of patients and the lack of randomization with the possible bias by propensity score matching. The efficacy of ischemia induction with RIPC was assessed clinically. Because the causes of death could not be ascertained for most of the deceased, mortality analysis in this study is restricted to all-cause mortality.

\section{Conclusions}

In conclusion, this study shows that TAVR associated systemic inflammation occurs frequently and is associated with an increased 6-month mortality. Our data do not lend support to the concept that RIPC reduces the occurrence of inflammatory response after TAVR.

\section{Author contributions}

KZ-manuscript preparation, data analysis, proof reading manuscript. WT-patient recruitment, remote ischemic preconditioning, data collection, data analysis, manuscript preparation, statistical analysis, proof reading manuscript. MK - statistical analysis. SW — study design, patient recruitment. $\mathrm{KI}-$ patient recruitment, TAVR procedure. $\mathrm{CP}$ - patient recruitment. $\mathrm{KMS}$ - patient recruitment, echocardiography. RW-computer tomography, proof reading manuscript. DF-statistical analysis, proof reading manuscript. TFR - proof reading manuscript. RHS — study design. AL_-proof reading manuscript. SQ and FMH contributed equally to this manuscript-study design, data analysis, manuscript preparation, proof reading manuscript. All authors read and approved the final manuscript.

\section{Ethics approval and consent to participate}

All subjects gave their informed consent for inclusion before they participated in the study. The study was conducted in accordance with the Declaration of Helsinki, and the protocol was approved by the Ethics Committee of TU Dresden (\# 73032013).

\section{Acknowledgment}

We would like to thank all patients participating in this study.

\section{Funding}

We sincerely thank the Technische Universität (TU) Dresden for granting the open access funds required for this publication (Open Access Funding by the Publication Fund of the TU Dresden).

\section{Conflict of interest}

TFR received honoraria as speaker, proctor, and/or preceptor from Abbott Laboratories and Edwards Lifesciences. All other authors declare no conflicts of interest.

\section{Supplementary material}

Supplementary material associated with this article can be found, in the online version, at https://www.imrpre ss.com/journal/RCM/23/1/10.31083/j.rcm2301020.

\section{References}

[1] Cribier A, Eltchaninoff H, Bash A, Borenstein N, Tron C, Bauer $\mathrm{F}$, et al. Percutaneous transcatheter implantation of an aortic valve prosthesis for calcific aortic stenosis: first human case description. Circulation. 2002; 106: 3006-3008.

[2] Kumar V, Sandhu GS, Harper CM, Ting HH, Rihal CS. Transcatheter Aortic Valve Replacement Programs: Clinical Outcomes and Developments. Journal of the American Heart Association. 2020; 9: e015921.

[3] Gorla R, Erbel R, Eagle KA, Bossone E. Systemic inflammatory response syndromes in the era of interventional cardiology. Vascular Pharmacology. 2018; 107: 53-66.

[4] Sinning JM, Scheer AC, Adenauer V, Ghanem A, Hammerstingl $\mathrm{C}$, Schueler R, et al. Systemic inflammatory response syndrome predicts increased mortality in patients after transcatheter aortic valve implantation. European Heart Journal. 2012; 33: 1459 1468.

[5] Lindman BR, Goldstein JS, Nassif ME, Zajarias A, Novak E, Tibrewala A, et al. Systemic inflammatory response syndrome after transcatheter or surgical aortic valve replacement. Heart. 2015; 101: 537-545.

[6] Kharbanda RK, Mortensen UM, White PA, Kristiansen SB, Schmidt MR, Hoschtitzky JA, et al. Transient limb ischemia induces remote ischemic preconditioning in vivo. Circulation. 2002; 106: 2881-2883.

[7] Heusch G, Botker HE, Przyklenk K, Redington A, Yellon D Remote ischemic conditioning. Journal of the American College of Cardiology. 2015; 65: 177-195.

[8] Konstantinov IE, Arab S, Kharbanda RK, Li J, Cheung MM, Cherepanov V, et al. The remote ischemic preconditioning stimulus modifies inflammatory gene expression in humans. Physiological Genomics. 2004; 19: 143-150. 
[9] Cai ZP, Parajuli N, Zheng X, Becker L. Remote ischemic preconditioning confers late protection against myocardial ischemia-reperfusion injury in mice by upregulating interleukin10. Basic Research in Cardiology. 2012; 107: 277.

[10] Otto CM, Baumgartner H. Updated 2017 European and American guidelines for prosthesis type and implantation mode in severe aortic stenosis. Heart. 2018; 104: 710-713.

[11] Luzzani A, Polati E, Dorizzi R, Rungatscher A, Pavan R, Merlini A. Comparison of procalcitonin and C-reactive protein as markers of sepsis. Critical Care Medicine. 2003; 31: 1737-1741.

[12] Ma L, Zhang H, Yin YL, Guo WZ, Ma YQ, Wang YB, et al. Role of interleukin-6 to differentiate sepsis from non-infectious systemic inflammatory response syndrome. Cytokine. 2016; 88: 126-135.

[13] Leon MB, Smith CR, Mack M, Miller DC, Moses JW, Svensson LG, et al. Transcatheter aortic-valve implantation for aortic stenosis in patients who cannot undergo surgery. The New England Journal of Medicine. 2010; 363: 1597-1607.

[14] Ho D, Imai K, King G, Stuart EA. MatchIt: Nonparametric Preprocessing for Parametric Causal Inference. Journal of Statistical Software. 2011; 42: 1-28.

[15] Rettig TCD, Nijenhuis VJ, Meek B, Rigter S, Ten Berg JM, Vlaminckx B, et al. Systemic Inflammation after Transcatheter Aortic Valve Implantation: A Prospective Exploratory Study. Journal of Cardiothoracic and Vascular Anesthesia. 2018; 32: e77-e82.

[16] Sinning JM, Ghanem A, Steinhauser H, Adenauer V, Hammerstingl C, Nickenig G, et al. Renal function as predictor of mortality in patients after percutaneous transcatheter aortic valve implantation. JACC: Cardiovascular Interventions. 2010; 3: 11411149.

[17] Bagur R, Webb JG, Nietlispach F, Dumont E, De Larochelliere $\mathrm{R}$, Doyle $\mathrm{D}$, et al. Acute kidney injury following transcatheter aortic valve implantation: predictive factors, prognostic value, and comparison with surgical aortic valve replacement. European Heart Journal. 2010; 31: 865-874.

[18] Nuis RJ, Van Mieghem NM, Tzikas A, Piazza N, Otten AM, Cheng J, et al. Frequency, determinants, and prognostic effects of acute kidney injury and red blood cell transfusion in patients undergoing transcatheter aortic valve implantation. Catheterization and Cardiovascular Interventions. 2011; 77: 881-889.

[19] Vincent JL, Opal SM, Marshall JC, Tracey KJ. Sepsis definitions: time for change. Lancet. 2013; 381: 774-775.

[20] Diegeler A, Doll N, Rauch T, Haberer D, Walther T, Falk V, et al. Humoral immune response during coronary artery bypass grafting: A comparison of limited approach, "off-pump" tech- nique, and conventional cardiopulmonary bypass. Circulation. 2000; 102: III95-100.

[21] Sablotzki A, Friedrich I, Muhling J, Dehne MG, Spillner J, Silber RE, et al. The systemic inflammatory response syndrome following cardiac surgery: different expression of proinflammatory cytokines and procalcitonin in patients with and without multiorgan dysfunctions. Perfusion. 2002; 17: 103-109.

[22] Franke A, Lante W, Fackeldey V, Becker HP, Kurig E, Zoller LG, et al. Pro-inflammatory cytokines after different kinds of cardiothoracic surgical procedures: is what we see what we know? European Journal of Cardio-Thoracic Surgery. 2005; 28: 569575.

[23] Asimakopoulos G. Mechanisms of the systemic inflammatory response. Perfusion. 1999; 14: 269-277.

[24] Kohsaka S, Menon V, Lowe AM, Lange M, Dzavik V, Sleeper LA, et al. Systemic inflammatory response syndrome after acute myocardial infarction complicated by cardiogenic shock. Archives of Internal Medicine. 2005; 165: 1643-1650.

[25] Hausenloy DJ, Yellon DM. Remote ischaemic preconditioning: underlying mechanisms and clinical application. Cardiovascular Research. 2008; 79: 377-386.

[26] Botker HE, Kharbanda R, Schmidt MR, Bottcher M, Kaltoft AK, Terkelsen CJ, et al. Remote ischaemic conditioning before hospital admission, as a complement to angioplasty, and effect on myocardial salvage in patients with acute myocardial infarction: a randomised trial. Lancet. 2010; 375: 727-734.

[27] Candilio L, Malik A, Ariti C, Barnard M, Di Salvo C, Lawrence $\mathrm{D}$, et al. Effect of remote ischaemic preconditioning on clinical outcomes in patients undergoing cardiac bypass surgery: a randomised controlled clinical trial. Heart. 2015; 101: 185-192.

[28] Thielmann M, Kottenberg E, Kleinbongard P, Wendt D, Gedik $\mathrm{N}$, Pasa S, et al. Cardioprotective and prognostic effects of remote ischaemic preconditioning in patients undergoing coronary artery bypass surgery: a single-centre randomised, double-blind, controlled trial. Lancet. 2013; 382: 597-604.

[29] Flechsig M, Ruf TF, Troeger W, Wiedemann S, Quick S, Ibrahim K, et al. Remote Ischemic Preconditioning Neither Improves Survival nor Reduces Myocardial or Kidney Injury in Patients Undergoing Transcatheter Aortic Valve Implantation (TAVI). Journal of Clinical Medicine. 2020; 9: 160.

[30] Kahlert P, Hildebrandt HA, Patsalis PC, Al-Rashid F, Janosi $\mathrm{RA}$, Nensa F, et al. No protection of heart, kidneys and brain by remote ischemic preconditioning before transfemoral transcatheter aortic valve implantation: Interim-analysis of a randomized single-blinded, placebo-controlled, single-center trial. International Journal of Cardiology. 2017; 231: 248-254. 